Revue d'histoire de l'Amérique française

ZWB REVUE D.HISTOIRE DE L'AMÉRIQUE FRANÇAISE

\title{
Réflexions sur la justice criminelle canadienne, au 18e siècle
}

\section{André Morel}

Volume 29, numéro 2, septembre 1975

URI : https://id.erudit.org/iderudit/303443ar

DOI : https://doi.org/10.7202/303443ar

Aller au sommaire du numéro

Éditeur(s)

Institut d'histoire de l'Amérique française

ISSN

0035-2357 (imprimé)

1492-1383 (numérique)

Découvrir la revue

Citer cette note

Morel, A. (1975). Réflexions sur la justice criminelle canadienne, au 18e siècle. Revue d'histoire de l'Amérique française, 29(2), 241-253.

https://doi.org/10.7202/303443ar d'utilisation que vous pouvez consulter en ligne.

https://apropos.erudit.org/fr/usagers/politique-dutilisation/ 


\title{
NOTE DE RECHERCHE
}

\section{RÉFLEXIONS SUR LA JUSTICE CRIMINELLE CANADIENNE, AU 18e SIËCLE *}

\author{
ANDré Morel \\ Faculté de droit \\ Université de Montréal
}

L'histoire de la justice criminelle constitue un champ d'investigation dont ne s'étaient guère préoccupés jusqu'à tout récemment les historiens. La responsabilité en revient peut-être d'ailleurs surtout aux historiens du droit qui, faute de s'être intéressés à l'histoire du droit pénal, rendaient pratiquement inaccessibles les archives des cours criminelles. On imagine sans peine le découragement de celui qui aborde un dépôt d'archives judiciaires sans avoir acquis préalablement une solide connaissance du vocabulaire technique, des degrés de juridiction et de leur compétence respective, de la marche de la procédure et de l'état du droit substantif à l'époque considérée. Et l'on conviendra que c'est bien à l'historien du droit, puisque c'est là sa compétence propre, qu'il appartient de fournir cette information. Sans elle, le chercheur abandonnera bien vite le dépouillement d'actes rédigés dans une langue trop hermétique; ou bien, s'il persiste malgré tout dans son travail, il se condamnera à coup sûr à des contresens et à de fausses interprétations qui enlèveront beaucoup de valeur aux résultats de ses recherches. Or, il n'y a guère plus d'une dizaine d'années que les historiens du droit, tant en France qu'au Québec, ont commencé à s'intéresser à l'histoire du droit pénal et à ouvrir ainsi un domaine de recherche tout nouveau qui se révèle déjà excessivement riche en enseignements de toutes sortes sur la société et les mentalités. Sous l'impulsion notamment de Pierre Chaunu, Yvonne Bongert, François Billacois et Bernard Schnapper, de grandes enquêtes sur la criminalité, dans la France de l'Ancien Régime, sont en cours et, dans cette même lignée, André Lachance a commencé à nous livrer des données précieuses sur

* Version remaniée d'un texte présenté à la réunion de la Société historique du Canada (Edmonton, juin 1975), en guise de commentaire à une communication d'André Lachance intitulée "Crimes, criminalité et répression pénale en Canada au 18e siècle (1712-1748)".

[241 ]

RHAF, vol. 29, no 2 (septembre 1975) 
la justice criminelle en Nouvelle-France. ${ }^{1}$ Les comparaisons que l'on peut faire entre ces données et celles qu'ont établies des recherches analogues portant sur la pratique judiciaire, soit métropolitaine, soit coloniale, m'amènent à présenter ici quelques réflexions. Les premières concernent la personnalité du criminel canadien de la première moitié du $18 \mathrm{e}$ siècle et les secondes, une des grandes catégories de crimes, ceux contre les mœurs. Ces brèves remarques n'ont pour but que d'illustrer la fécondité de ces récentes enquêtes historiques et de suggérer encore d'autres voies aux recherches futures.

\section{I - LA PERSONNALITÉ DU CRIMINEL CANADIEN}

Par opposition à la procédure criminelle anglaise qui est essentiellement orale et qui laisse par conséquent peu de traces de son déroulement, la procédure criminelle française de l'Ancien Régime avait cette caractéristique d'être une procédure écrite dont la marche était minutieusement réglée par l'ordonnance criminelle du mois d'août 1670. Tout, depuis la plainte jusqu'au jugement définitif, devait être consigné par écrit. Et notamment un greffier devait tenir un procès-verbal fidèle des informations, puis de l'interrogatoire de l'accusé, du récolement et de la confrontation de l'accusé et des témoins. C'est évidemment pour l'historien un avantage exceptionnel, puisqu'il peut ainsi, du moins lorsque les dossiers de l'enquête et du procès ont été conservés jusqu'à nous, reconstituer le portrait des accusés: leur état civil, leur profession, leur milieu de vie et même certains traits de leur personnalité. Ainsi André Lachance a-t-il pu dresser des fiches plus ou moins complètes sur chacun des quelque 800 délinquants qu'il a rencontrés pour présenter le portrait collectif du criminel. De tous les renseignements que ces fiches comportent, j'en retiens ici deux : le sexe et l'âge.

\section{$1^{\circ}$ - Le sexe}

L'immense majorité de ces délinquants canadiens est constituée d'hommes. Et c'est bien le contraire qui nous aurait étonnés, car la criminalité d'aujourd'hui est, elle aussi, une criminalité principalement masculine. Peut-être l'est-elle d'ailleurs davantage

1 Voir sa thèse de doctorat, "La justice criminelle du roi en Canada, 1712-1748" (Université d'Ottawa, 1974) ; voir aussi l'article "Le Bureau des Pauvres de Montréal 1698-1699: contribution à l'étude de la société montréalaise de la fin du 17e siècle", Histoire sociale (novembre 1969): 100-110. 
que dans la société du 18e siècle, car la part des femmes dans le groupe humain étudié par M. Lachance, tout minoritaire qu'elle soit, s'élève tout de même à $21 \%$. C'est une proportion tout à fait identique qu'a relevée $M$. Petrovitch dans son étude sur la criminalité à Paris, dans la seconde moitié du 18e siècle (320 femmes sur 1533 accusés). ${ }^{2}$ Or, les statistiques sur la criminalité au Canada à l'heure actuelle nous indiquent que la proportion des femmes condamnées est, de nos jours, sensiblement plus faible. ${ }^{3}$ L'explication de ce fait n'est pas nécessairement aisée ni simple. Mais si on voulait s'y arrêter, il faudrait tout d'abord distinguer le degré de participation des femmes dans les diverses espèces de crimes. Car il existe "une certaine spécialisation par sexe dans le crime". Dans sa thèse, André Lachance a relevé en effet que la criminalité féminine atteint son sommet - près de $30 \%$ dans les crimes contre les biens. Il y a une femme accusée de vol pour deux hommes; et en matière de recel, les femmes sont presque aussi nombreuses que les hommes $(43,5 \%)$. Mais cela ne paraît pas être le propre de la société canadienne: les recherches menées en France pour la même époque aboutissent aux mêmes constatations. ${ }^{4}$ Il est donc vraisemblable que l'importance de la criminalité féminine, au $18 \mathrm{e}$ siècle, soit attribuable à certains traits de la société d'Ancien Régime qui se retrouvent de part et d'autre de l'Atlantique. Pour les identifier, il faudrait pousser l'analyse des statistiques criminelles plus loin qu'on ne l'a fait jusqu'à maintenant. Il importerait également d'interpréter avec soin un certain nombre de dossiers individualisés pour faire l'équivalent de ce que les médecins appellent des "histoires de cas".

Un autre problème qui est lié au sexe des condamnés est celui de la gravité des peines qui sont infligées aux hommes et aux femmes respectivement. Les juges canadiens auraient-ils fait montre de moins de rigueur à l'égard des femmes que des hommes dans le choix des châtiments imposés? Si nous rapportons le nombre de condamnations, exprimé en pourcentage, prononcées contre les femmes au taux de la criminalité féminine, il faut

2 Porphyre Petrovitch, "Recherches sur la criminalité à Paris dans la seconde moitié du XVIIIe siècle", dans A. Abbiateci et al., Crimes et criminalité en France sous l'Ancien Régime, 17e-18e siècles, Cahiers des Annales (Paris, Armand Colin, 1971), 234.

3 En 1967, les femmes ne représentaient que $12,7 \%$ de tous les individus condamnés pour des actes criminels, dans l'ensemble du Canada. Pour le Québec, ce taux n'était même que de 11,5\%. Voir Bureau fédéral de la statistique, Statistique de la criminalité, 1967 (Ottawa, Imprimeur de la Reine, 1969), tableau 2, p. 32-33, et tableau 5, p. 40-43.

4 P. Petrovitch, op. cit., 235. 
nuancer la réponse (tableau A). Nous constatons que, compte tenu du nombre des délinquantes par rapport à l'ensemble des criminels, les femmes ont été condamnées à la peine capitale aussi souvent que les hommes; mais en revanche on n'a prononcé qu'assez rarement contre elles les peines du fouet et du carcan. Le blâme et l'admonestation, peines infamantes relativement bénignes, ne sont pas non plus considérées comme un traitement de faveur pour les femmes, puisque, proportionnellement à leur nombre, autant d'hommes ont "bénéficié" des mêmes peines. Par contre, pour certaines peines, il y a des disparités notables: comme pour le fouet et le carcan et au même degré qu'eux d'ailleurs, le bannissement est en pratique une peine à caractère nettement masculin; tandis que l'amende honorable apparaît comme un châtiment pour lequel les tribunaux canadiens témoignent d'une prédilection bien marquée lorsque le coupable est une femme. Peut-être cela tient-il au fait que l'amende honorable est considérée comme une peine corporelle grave sans pour autant porter atteinte à l'intégrité physique du condamné. Serait-ce là, chez nos juges hommes du 18e siècle, une sensibilité à l'égard de la femme? Notons enfin que la réclusion dans un Hôpital général est la seule peine qui soit de droit réservée aux femmes comme substitut de la peine des galères à laquelle seuls les hommes peuvent être condamnés. Mais on était plus prompt, semble-t-il, à enfermer les femmes dans une cellule qu'à expédier les hommes aux galères. Il est vrai que le sort des premières devait paraître plus supportable que celui des galériens.

Si mes observations sont exactes, on constaterait donc un traitement égalitaire entre les sexes dans l'imposition des châtiments les plus sévères (la mort) comme les plus légers (le blâme et l'admonestation). Mais entre ces deux extrémités de l'échelle des peines, entre les crimes les plus graves qui méritent la mort et les délits bénins qui n'entraînent qu'une réprimande, nos tribunaux auraient, selon toute apparence, adopté une attitude discriminatoire suivant la nature de la peine et le sexe du coupable. Si des recherches ultérieures devaient confirmer l'existence d'une telle attitude de la part de nos juges, il faudrait à coup sûr s'interroger plus avant pour en découvrir les motifs profonds.

\section{$2^{\circ}$ - L'âge}

M. Lachance a aussi relevé des renseignements concernant l'âge des délinquants et constaté que l'âge de la délinquance maximum se situe entre 20 et 29 ans. 
TABLEAU A

Peines et criminalité féminine en Nouvelle-France, $1712-1748^{*}$

\begin{tabular}{|l|c|c|c|c|}
\hline \multicolumn{1}{|c|}{$\begin{array}{c}\text { Peines } \\
\text { par ordre } \\
\text { de gravité }\end{array}$} & $\begin{array}{c}\text { Nombre total } \\
\text { des condam- } \\
\text { nations }\end{array}$ & $\begin{array}{c}\text { Nombre } \\
\text { de femmes } \\
\text { condamnées }\end{array}$ & $\begin{array}{c}\text { Rapport au } \\
\text { Pourcentage } \\
\text { des femmes } \\
\text { condamnées }\end{array}$ & $\begin{array}{c}\text { criminaliá } \\
\text { féminine } \\
\text { (21\%) }\end{array}$ \\
\hline $\begin{array}{l}\text { 1. Mort } \\
\begin{array}{l}\text { 2. Galères (hommes) } \\
\text { et réclusion }\end{array}\end{array}$ & 38 & 8 & $21 \%$ & $=$ \\
$\begin{array}{l}\text { (femmes) } \\
\text { 3. Bannissement }\end{array}$ & 38 & 11 & $29 \%$ & + \\
$\begin{array}{l}\text { 4. Fouet } \\
\text { 5. Amende } \\
\text { honorable }\end{array}$ & 39 & 6 & $13 \%$ & - \\
6. Carcan & 20 & 9 & $13 \%$ & - \\
7. Blâme et & 8 & 1 & $12,5 \%$ & - \\
admonestation & 54 & 12 & $22 \%$ & $=$ \\
\hline
\end{tabular}

* Les données numériques ont été tirées du tableau $\mathrm{X}$ de la communication d'A. Lachance citée plus haut.

Il faudrait toutefois cerner de plus près l'âge auquel se situent les records de délinquance. Bien sûr l'âge que les accusés déclarent avoir, au cours des interrogatoires, est souvent approximatif; mais on considère généralement que, sur l'ensemble, les inexactitudes se compensent. Il importerait de regrouper les accusés non plus par classe d'âge de cinq ans en cinq ans, mais d'année en année. Les données actuelles de la criminologie nous enseignent en effet d'une part qu'il peut y avoir des variations sensibles du taux de délinquance entre les individus qui font partie du groupe des 20 à 30 ans; et d'autre part que les records de délinquance ne se retrouvent pas nécessairement au même âge chez les hommes et chez les femmes. Il serait étonnant qu'il en ait été autrement pour la société canadienne de la première moitié du $18 \mathrm{e}$ siècle. Il faudrait donc établir d'abord la pyramide d'âge 
des accusés pour la période 1712-1748, en distinguant entre les hommes et les femmes. Peut-être ne serait-il pas alors sans intérêt de comparer cette pyramide non seulement à celles qui ont été établies pour certaines régions de la France du $18 \mathrm{e}$ siècle, ${ }^{5}$ mais aussi à la pyramide d'âge des accusés de la société québécoise contemporaine. De telles comparaisons ne manqueraient certainement pas de susciter des questions et de stimuler la recherche.

Par ailleurs, si on s'en tient aux statistiques fournies par A. Lachance, il ressort que nous avons une apparition brusque et importante de la criminalité entre 15 et 19 ans, un palier assez élevé entre 30 et 44 ans, et une décroissance lente, mais à peu près régulière de la criminalité chez les plus âgés. Mais ces nombres absolus sont trompeurs. Pour leur donner une signification réelle, il faudrait mettre en parallèle la population délinquante et la population globale en tenant compte toujours de la répartition par classes d'âge. Les études de démographie historique, depuis celles de Jacques Henripin, commencent à nous donner une image assez précise de ce qu'était la pyramide démographique du Canada, au 18e siècle. En établissant des rapports entre les différentes classes d'âge des criminels et celles de la population globale, on discernerait sans doute, pour certaines catégories d'âge, des baisses ou des hausses notables de délinquance. On s'apercevrait peut-être que la délinquance des jeunes dans la vingtaine - qui représentent $40 \%$ de tous les criminels dont l'âge nous est connu - est une délinquance normale compte tenu de la place considérable que les individus de cet âge occupent dans l'ensemble de la population de la colonie. Et, à l'inverse, les 13 sexagénaires condamnés qu'a recensés A. Lachance pourraient bien révéler une recrudescence de la criminalité chez les vieillards qui sont, à cette époque, bien peu nombreux dans l'ensemble de la population.

Enfin, le phénomène de la délinquance juvénile mériterait à lui seul une étude approfondie. Le nombre des jeunes traduits devant les tribunaux criminels, entre 1712 et 1748 , est en effet assez considérable pour autoriser une recherche spécifique. Mais le premier problème à trancher sera celui de la définition du jeune délinquant: à partir de quel âge le délinquant doit-il être considéré comme un adulte? Il est vrai que, par emprunt au droit romain, la minorité de l'ancien droit était fixée à 25 ans; mais, à l'intérieur de cette catégorie des moins de 25 ans, le régime de responsabilité pénale variait considérablement selon l'âge du

5 Voir, par exemple, P. Petrovitch, op. cit., 236. 
mineur. Les criminalistes du $18 \mathrm{e}$ siècle, quant à eux, enseignaient que la fille jusqu'à $91 / 2$ ans et le garçon jusqu'à $101 / 2$ ans étaient irresponsables, mais qu'au-delà le mineur, surtout s'il n'était pas encore pubère ou ne l'était que depuis peu, devait être traité moins sévèrement que le majeur. Toutefois entre les distinctions de la doctrine et la pratique des tribunaux, il semble bien y avoir eu un écart assez large. Pour les juges, aucun âge déterminé n'opérait de partage réel bien net entre ceux que l'on traitait pénalement comme des majeurs et ceux qui bénéficiaient d'une responsabilité atténuée. ${ }^{6}$ Dans ces conditions, on peut se demander si, pour définir le jeune délinquant, on ne doit pas adopter la classification de la législation actuelle qui, au Québec comme dans quelques autres provinces canadiennes, fixe la majorité pénale à 18 ans. $^{7}$ Cette solution qui n'est pas à l'abri de tout reproche, offre du moins l'avantage de faciliter les comparaisons entre le jeune délinquant de l'Ancien Régime et celui d'aujourd'hui.

Quelles sont les caractéristiques de cette délinquance juvénile? Dans sa thèse, $M$. Lachance montre que les mineurs de 10 à 19 ans auraient été accusés deux fois plus souvent de crimes contre les personnes que de crimes contre les biens. En cela, le jeune délinquant canadien a un comportement sensiblement différent de son homonyme parisien étudié par Mlle Bongert pour le $18 \mathrm{e}$ siècle, qui, lui, est presque exclusivement voleur, filou et escroc. ${ }^{8}$ Il est vrai que la criminalité parisienne de cette époque est, elle aussi, à $87 \%$ une criminalité du vol: ${ }^{9}$ le criminel en herbe n'est donc pas, à Paris, distinct de son aîné. Mais ce quasi-monopole du vol semble être un trait des grandes agglomérations urbaines. Chez nous, les voies de fait et les délits plus graves contre les personnes représentent de loin les crimes les plus nombreux $(67 \%)$. Pour trois accusés de cette catégorie de crimes, il n'y en a qu'un accusé de vol et larcin $(20 \%)$. Le mineur canadien manifesterait donc une tendance légèrement plus marquée que l'adulte à s'accaparer le bien d'autrui, mais il serait aussi souvent que lui impliqué dans les querelles de voisins, les rixes et les propos injurieux.

${ }^{6}$ Yvonne Bongert, "Délinquance juvénile et responsabilité pénale du mineur au XVIIIe siècle", dans A. Abbiatici et al., op. cit., 70 et ss.

${ }^{7} \mathrm{La}$ Loi sur les jeunes délinquants (Statuts revisés du Canada 1970, chapitre J-3, art. 2) s'applique à l'enfant de moins de 16 ans, mais permet d'élever cet âge jusqu'à 18 ans par des mesures d'application provinciale. Voir aussi les art. 12 et 13 du Code criminel.

$8 \mathrm{Y}$. Bongert, op. cit., 58.

9 P. Petrovitch, op. cit., 208. 
Qui est d'ailleurs au juste ce délinquant? Majoritairement, c'est un garçon assurément. Mais le pourcentage de la délinquance juvénile féminine est-il aussi élevé que chez les adultes? Ou bien l'attirance des femmes pour le crime se manifeste-t-elle plus tardivement que chez les garçons; et s'il en est ainsi, à quel âge se produit ce changement? D'après l'étude de Mlle Bongert pour le $18 \mathrm{e}$ siècle à Paris, la criminalité culmine à 15 ans chez les garçons, tandis qu'elle n'atteint son sommet qu'à 17 ans chez les filles ${ }^{10}$. En allait-il autrement chez nous? Il faudrait enfin essayer de comprendre, par un examen minutieux de la jurisprudence, suivant quels critères nos juges arrêtaient la peine des jeunes condamnés: tenaient-ils compte de l'âge, du sexe du coupable, des possibilités de sa prise en charge par ses parents ou des amis? Cherchaient-ils, de façon plus ou moins confuse et avec les moyens dont ils disposaient, la correction de l'enfant et son redressement plutôt que son châtiment?

On pourrait continuer de multiplier les questions pour lesquelles on ne connaît pas pour le moment les réponses. Il y a là pourtant bien des avenues à explorer, mon propos n'étant que de les suggérer.

\section{II - LES CRIMES CONTRE LES MOEURS}

André Lachance n'a pas étudié spécifiquement cette catégorie de crimes qu'on appelle les crimes contre les mœurs, mais on trouve, dans sa thèse, bon nombre de remarques et de données statistiques qui m'ont incité à essayer de pousser l'analyse un peu plus loin.

Si on n'inclut, comme il se doit, dans la catégorie des crimes contre les mœurs que les seules infractions à la morale sexuelle que le droit français du $18 \mathrm{e}$ siècle considérait comme des comportements criminels et punissait à ce titre, on ne relève ${ }^{11} \mathrm{au}$ total, pour la période étudiée par A. Lachance, que 26 procès de cette catégorie, impliquant 47 accusés. C'est à peine $5 \%$ de toutes les affaires criminelles entre 1712 et 1748 ; et $6 \%$ de tous les accusés.

Même si ce taux de $5 \%$ est trois fois plus élevé que celui des affaires de mœurs jugées par le Châtelet de Paris, dans la

10 Y. Bongert, op. cit., 62-64.

11 Ce recensement a été fait à partir du "relevé nominatif des criminels canadiens entre 1712 et 1748 " établi par A. Lachance, dans sa thèse, appendice $\mathrm{A}$. 
deuxième moitié du $18 \mathrm{e}$ siècle ${ }^{12}$, il reste malgré tout fort modeste. C'est en tout cas, par le nombre des affaires, la moins importante des quatre grandes catégories d'actes criminels, passant même après les crimes contre l'autorité publique ou contre l'Etat.

Non seulement nos tribunaux criminels n'ont-ils pas eu souvent l'occasion de réprimer des attentats aux mœurs, mais parmi la longue liste de crimes dits "de luxure" qu'énumèrent les criminalistes, il en est plusieurs qui ne sont pas même représentés au cours de la période 1712-1748: il en est ainsi en particulier du viol, de l'inceste, de la sodomie, de la bestialité et de la fornication.

Par ailleurs, A. Lachance a noté un fait surprenant et qui reste pour l'instant inexpliqué: la baisse constante et fort prononcée du nombre des inculpations criminelles tout au long de la période étudiée, alors qu'on se serait attendu à une tendance inverse étant donné l'accroissement notable de la population au cours de cette même période. Tandis que la population augmente de $125 \%$, note $\mathrm{M}$. Lachance, le taux des accusations diminue de $75 \%$. Pour ce qui est des seuls crimes de mœurs, non seulement faut-il remarquer une diminution parallèle du nombre des accusations au long des années, mais il se produit même un déclin relatif encore plus marqué que celui qui touche l'ensemble des procès criminels. En découpant en effet la période étudiée en trois tranches de douze années chacune, on constate tout d'abord une chute régulière du nombre des affaires de mœurs instruites devant les tribunaux, le nombre de ces affaires passant de 13 pour les années 1712-1723, à 5 seulement pour les années 17361748 (tableau B).

Or, si on met en rapport le nombre des affaires de mœurs instruites et le nombre total des inculpations pour chacune des périodes de douze années, on s'aperçoit que le pourcentage des affaires de mœurs qui représentait 6,4\% de l'activité judiciaire en 1712-1723, s'abaisse à $4,2 \%$ en $1724-1735$, pour finalement tomber à $3,9 \%$ en 1736-1748.

Toutes ces constatations - le nombre presque insignifiant de poursuites pour crimes contre les mœurs, l'absence d'inculpations pour plusieurs crimes de cette catégorie, enfin le déclin constant dans la fréquence des procès de ce type - tout cela forme un faisceau d'indications concordantes que personne n'aura la naïveté d'interpréter comme étant une preuve de progrès continu dans la vertu et la moralité des Canadiens...

${ }^{12}$ Les affaires de mœurs ne représentent que $1,6 \%$ des procès parisiens, selon P. Petrovitch, op. cit., 208. 
TABLEAU B

Crimes contre les mœurs

par rapport au total des crimes

\begin{tabular}{|c|c|c|c|c|}
\hline Années & $1712-1723$ & $1724-1735$ & $1736-1748$ & Total \\
\cline { 2 - 4 } & 13 & 8 & 5 & 26 \\
$\begin{array}{c}\text { Crimes } \\
\text { de } \\
\text { meurs }\end{array}$ & 203 & 189 & 127 & 519 \\
\hline $\begin{array}{c}\text { Total } \\
\text { des } \\
\text { crimes }\end{array}$ & $6,4 \%$ & $4,2 \%$ & $3,9 \%$ & $5 \%$ \\
\hline$\%$ & & \\
\hline
\end{tabular}

Pour éclairer les données que l'enquête d'A. Lachance a révélées, il serait évidemment bien utile de pouvoir disposer de renseignements aussi précis pour le $17 \mathrm{e}$ siècle. Mais à défaut d'une telle recherche, on peut peut-être, à titre indicatif du moins, considérer ce que l'on connaît de la répression criminelle à Montréal entre 1648 et 1693 , étant entendu que nous ne possédons plus tous les dossiers du tribunal montréalais et qu'une proportion indéterminée de documents n'a pas été conservée jusqu'à nous ${ }^{13}$. De ce qui subsiste cependant, nous ne pouvons qu'être frappés par le pourcentage étonnamment élevé d'affaires de mœurs: un quart de tous les procès criminels à l'époque de Maisonneuve $(24 \%: 4$ sur 17$)$, un cinquième $(19 \%: 12$ sur 63$)$ de ceux dont a été saisi le baillage entre 1663 et 1693. Au surplus, presque chacune des espèces d'atteintes aux mœurs comprises dans ce qu'on dénommait "crimes de luxure" est représentée dans la pratique montréalaise de la seconde moitié du $17 \mathrm{e}$ siècle: adultère $(2$ cas $)$, bigamie, $(2$ cas $)$, inceste $(1$ cas $)$, viol et tentative de viol ( 3 cas $)$, rapt et séduction ( 2 cas), attentat à la pudeur de jeunes enfants (1 cas), concubinage (2 cas), libertinage ( 1 cas), et même la sodomie ( 2 cas) dont les criminalistes

$13 \mathrm{~J}$ 'utilise ici les constatations faites au cours de recherches personnelles sur la justice criminelle à Montréal au 17e siècle et dont les résultats n'ont été publiés qu'en partie: A. Morel, "L'imposition et le contrôle des peines au Bailliage de Montréal (1666-1693)", Etudes juridiques en hommage $\grave{a}$ Monsieur le juge Bernard Bissonnette (Montréal, Presses de l'Université de Montréal, 1963), 413-432. 
de l'Ancien Régime nous disent qu'il n'arrive que très rarement que ce crime soit porté à la connaissance des juges.

On le voit, en matière d'atteinte aux mœurs, la répression criminelle offre un tableau fortement contrasté. Il n'y a pas de raison de croire que le décalage très net que l'on remarque dans la répression des affaires de mœurs entre le $17 \mathrm{e}$ et le $18 \mathrm{e}$ siècles soit attribuable à un appareil policier qui serait devenu avec le temps moins efficace. Et il est certain par ailleurs que la criminalité réelle, dans ce type d'affaires, loin de décroître, a dû au contraire augmenter dans les faits. Ce que nous constatons, c'est une diminution constante et appréciable, entre le milieu du $17 \mathrm{e}$ et le milieu du 18 e siècles, de la criminalité légale, c'est-à-dire du nombre de délinquants sexuels qui ont été effectivement l'objet de poursuites judiciaires. Ce fait pourrait bien être le signe d'une modification importante dans l'échelle des valeurs de la société. Sans qu'il y ait pour autant une plus grande dépravation des mœurs, on est porté à croire que c'est parce que les infractions à la morale sexuelle deviennent de moins en moins source d'indignation et de scandale qu'elles sont en conséquence de plus en plus rarement dénoncées à la justice. N'est-il pas d'ailleurs à cet égard significatif que le délit contre les mœurs qui est, et de beaucoup, le plus souvent porté à la connaissance des tribunaux, au 18e siècle, soit le crime de rapt et de séduction: 17 affaires sur 26. Or, dans ces affaires, la victime est le plus souvent une fille mineure, quelquefois une domestique. Ce qui est alors en jeu, ce n'est pas tant la pureté morale de la victime que l'atteinte portée à l'autorité du père sur son enfant ou du maître sur son domestique. Cette interprétation, en amenuisant encore davantage le nombre des véritables affaires de mœurs, viendrait confirmer notre hypothèse.

Reste alors à s'interroger sur l'attitude des juges canadiens, lorsqu'ils sont appelés à connaître d'une affaire de mœurs. La plus ou moins grande sévérité de la justice peut en effet être une indication des valeurs réelles que l'on cherche à protéger. Or, une comparaison rapide des peines infligées aux accusés trouvés coupables de crimes contre les mœurs fait bien vite ressortir, de la part des juges du $17 \mathrm{e}$ siècle, une sévérité dont on n'a plus guère de manifestation au siècle suivant. Le bannissement plus souvent perpétuel qu'à temps - est la peine qui est presque invariablement retenue, au $17 \mathrm{e}$ siècle, pour tous les crimes autres que le rapt; au 18e siècle, on ne le prononce plus que contre 
l'homme adultère. Le concubinage ne semble guère troubler la conscience des juges et les poursuites tournent court, trois fois sur quatre, sans qu'aucune condamnation ne soit portée. Ceux qui comparaissent pour rapt ou séduction s'en tirent généralement avec une interdiction de fréquenter la fille qu'ils ont cherché à enlever ou à séduire, quand ce n'est pas par une mise hors de cour. Il n'en va autrement que lorsque la jeune fille mineure est enceinte, auquel cas le ravisseur est chargé du soin de s'occuper de l'enfant à naître, à moins qu'il ne préfère épouser la fille. Dans un seul cas de rapt, le coupable a été condamné aux galères à perpétuité, mais il n'est pas indifférent de remarquer qu'il s'agissait d'un esclave panis.

Les manquements à la foi conjugale sont toujours punis avec la même rigueur, que ce soit au $18 \mathrm{e}$ siècle ou à l'époque de Maisonneuve: l'homme adultère est banni, la femme enfermée dans une maison de force pour le reste de ses jours et déchue de tous ses avantages matrimoniaux. Les actes d'attentat à la pudeur commis sur de jeunes enfants sont, eux aussi, jugés très sévèrement: en 1683, un individu coupable d'actes indécents sur des fillettes est banni à perpétuité; cinquante ans plus tard, un soldat de 60 ans est même condamné à mort pour avoir eu une pareille conduite envers une petite fille de 6 ans. Contre les filles publiques, on sévit occasionnellement. On les arrête, par groupe de 2,3 ou 4 , et on les condamne à la peine la plus grave qui se puisse prononcer contre des prostituées: la réclusion dans un Hôpital général. Mais une fois cette opération de salubrité publique effectuée qui devait apaiser la conscience populaire, il se passe plusieurs années avant qu'on ne répète le même scénario.

Cette justice est, à certains égards, une justice qui nous déconcerte. Elle est d'abord fort éloignée de celle que nous présentent les criminalistes du temps qui n'ont pas de mots assez forts pour qualifier les crimes de luxure et qui ne manquent pas de brandir les supplices les plus cruels contre les coupables. Mais la pratique judiciaire canadienne ne fait pour ainsi dire jamais montre d'une pareille rigueur dans les châtiments. Certes, les affaires de mœurs heurtent davantage l'opinion publique au $17 \mathrm{e}$ qu'au 18e siècle, mais les juges, même au $17 \mathrm{e}$ siècle, répugnent à infliger des peines cruelles, fut-ce le fouet. La société canadienne du $17 \mathrm{e}$ siècle ne cherche pas tant à se venger qu'à se débarrasser de ceux qui violent les valeurs morales auxquelles elle croit. 
Enfin, cette justice nous apparaît, au 18e siècle, comme une justice qui ne s'indigne plus, comme elle le faisait encore au siècle précédent, de certains manquements à la morale sexuelle. Elle ne se donne même plus la peine de traduire devant les tribunaux ceux qui enfreignent certains tabous. Elle est pleine d'indulgence pour les déviants sexuels en général, mais elle retrouve toute sa rigueur passée, lorsque c'est la famille qui est perturbée, comme dans le cas d'adultère, ou lorsque ce sont de jeunes enfants qui sont les victimes. 